\title{
A DILUTION TEST FOR THE CONVERGENCE OF SUBSERIES OF A MONOTONE SERIES
}

\section{LASSE LESKELÄ AND MIKKO STENLUND}

Abstract. Cauchy's condensation test allows to determine the convergence of a monotone series by looking at a weighted subseries that only involves terms of the original series indexed by the powers of two. It is natural to ask whether the converse is also true: Is it possible to determine the convergence of an arbitrary subseries of a monotone series by looking at a suitably weighted version of the original series? In this note we show that the answer is affirmative and introduce a new convergence test particularly designed for this purpose.

Mathematics subject classification (2010): 40A05.

Keywords and phrases: condensation test, dilution test, subseries, thinning, polynomially sparse subsequence, counting function.

\section{REFERENCES}

[1] C. R. BanerJee And B. K. LahiRi, On subseries of divergent series, Amer. Math. Monthly, 71:767$768,1964$.

[2] G. H. Behforooz, Thinning out the harmonic series, Math. Mag., 68(4):289-293, 1995.

[3] D. D. Bonar And M. Khoury, Jr., Real Infinite Series, Mathematical Association of America, Washington DC, 2006.

[4] G. H. Hardy And E. M. Wright, An Introduction to the Theory of Numbers, Oxford University Press, sixth edition, 2008.

[5] A. J. Kemper, A curious convergent series, Amer. Math. Monthly, 21(2):48-50, 1914.

[6] T. Schmelzer AND R. BAillie, Summing a curious, slowly convergent series, Amer. Math. Monthly, 115(6):525-540, 2008. 